\section{BIG FAT CAMP}

There are many depressing things about the current epidemic of childhood obesity. One of these is that most interventions tested to date simply do not work. As Norway has some of the lowest obesity rates in international comparisons there will be those who wonder whether an intervention trial conducted there has generalisability; but the findings, yet again, that a plausible and rational approach to treatment has no benefit over conventional outpatient management are all too likely to be generally true. As Benestad et al report, the Camp-based Family Treatment of Childhood Obesity was intensive, prolonged ( 2 years) and multi-modal, so it was also expensive: another example of how important it is to evaluate plausible ideas rigorously, and publish the negative results, so that cash-strapped services do not waste money on ineffective treatments. See page 303.

\section{WIN-WIN FOR IMMUNISATION}

If obesity is professionally depressing, then one thing to lighten the mood should be immunisation, one of the great medical triumphs of recent times. Garon and Patel explain developments in polio immunisation world-wide. As wild-type polio has virtually disappeared, the tiny proportion of cases associated with the live oral vaccine has become much more important, and is addressed by switching to the injectable vaccine. They also describe other interesting nuances to the polio programme. Closer to home for many readers is rotavirus immunisation in infancy, for which Forrest et al chart the impact of the programme around Lothian in Scotland: it works well in the target age group. Encouragingly they provide evidence that the programme has conferred substantial protection for older children who were not immunised but seem to have benefited from the programme indirectly. See pages 323 and 362 .

\section{ATTENTION DEFICIT HYPERACTIVITY DISORDER: TOO MUCH DIAGNOSIS OR TOO LITTLE?}

Attention deficit hyperactivity disorder (ADHD) generates polarised opinions, conflicting narratives, and a great deal of hot air. There are those who argue that it does not even exist, for example $A D H D$ Does Not Exist by Richard Saul (HarperCollins 2014), and there is some evidence that a substantial proportion of children with this label are misdiagnosed. So what is the truth, or is 'truth' too slippery a word in a world of alternative facts? We try to insert a little balance into the debate by carrying Professor Eric Taylor's review of the subject. The 'real' prevalence as determined by good quality research seems to be around $5 \%$ of the child population, so the fundamental premise of the review is not only that ADHD does exist, but in the UK at least it is probably under-diagnosed. See page 376.

\section{JOINT DIAGNOSIS}

When a child presents with a hot red joint, establishing whether or not they have septic arthritis is regarded as the crucial first step. But it is not always so easy to do, and what is perhaps less well known is that misdiagnosis matters both ways: harm to the joint can just as easily occur if a child is treated for infection, but actually has juvenile idiopathic arthritis. Unfortunately, as Aupiais et al show, measures conventionally used to attempt to distinguish infection from non-infection such as c-reactive protein, parameters of the full blood count, and cell counts in synovial fluid, lack specificity and overlap substantially between the two conditions. Put simply, we have no satisfactory tests at present by which to achieve timely and accurate diagnosis. It is important that we recognise this rather than pretend otherwise. See page 316.

\section{A VERY RARE DISEASE}

Ataxia telangiectasia is one of those rare diseases that we all mug up ahead of examinations, but which in practice we are most unlikely to encounter as the estimated prevalence according to Orphanet is probably less than 1 in 100000 children. Consequently, as Devaney et al point out, there is a wide range in the age at diagnosis, with the likelihood that in some cases diagnosis may be subject to excessive delay. The key test to be done if ataxia-telangiectasia is in a differential diagnosis is the serum alpha-fetoprotein concentration. The authors note that ataxia telangiectasia provides a paradigm for other very rare diseases and their diagnosis. They refer to an editorial on the problem of rare diseases which we published in 2011; in 2013 the Department of Health published the UK Strategy for Rare Diseases (https://www.gov.uk/ government/collections/rare-diseases) and Public Health England is developing a rare diseases register (declaration of interest: I am the Clinical Lead for the National Congenital Anomaly and Rare Diseases Registration Service, or NCARDRS). We have commissioned a piece on progress with NCARDRS that we hope to publish later this year. See page 323.

\section{ADRENAL SUPPRESSION FROM GLUCOCORTICOID TREATMENT}

I can't recall seeing recent, reliable data on the prevalence of long term glucocorticoid treatment in children, or indeed a figure for what proportion of children receive a short course of systemic glucocorticoid treatment every year. If these prevalences and proportions have risen over the years, maybe that represents more children getting appropriate treatment; certainly the 'steroid phobia' that was widespread when I was a junior doctor seems to have faded away. So what about the other side of the coin: how many children are harmed or potentially harmed by adrenal suppression secondary to glucocorticoid exposure? Using the Canadian Paediatric Surveillance Program, Goldbloom et al ascertained the minimum annual incidence up to 18 years: 0.35 per 100000 children. Clearly the incidence in the subpopulation of children exposed to longterm steroid treatment will be considerably greater. They remind us that corticosteroid toxicity is often an avoidable harm and that to prevent it requires constant vigilance. An accompanying Editorial by Bayman and Drake amplifies these points and reminds us that individual children differ considerably in their sensitivity to glucocorticoid toxicity, so it is important not to be complacent about dosages that do not seem all that high. See pages 340 and 338 . 